Canad. Math. Bull. Vol. 22 (2), 1979

\title{
ON BERNDT'S METHOD IN ARITHMETICAL FUNCTIONS AND CONTOUR INTEGRATION
}

\author{
BY \\ P. V. KRISHNAIAH AND R. SITA RAMA CHANDRA RAO
}

Introduction. If $f$ is a suitable meromorphic function then, by a classical technique in the calculus of residues, one can evaluate in closed form series of the form

$$
\sum_{n=-\infty}^{\infty} a(n) f(n) \text { or } \sum_{n=-\infty}^{\infty}(-1)^{n} f(n)
$$

Recently Bruce C. Berndt ([1]) considered using contour integration for the evaluation of series of the form

$$
\sum_{n=-\infty}^{\infty} f(n) \text { or } \sum_{n=-\infty}^{\infty}(-1)^{n} a(n) f(n) .
$$

where $f$ belongs to a suitable class of rational functions and $a(n)$ is an arithmetical function. He developed a new method to transform series of type (1.2) above (and of slightly more general types) into series generally involving a different arithmetical function. As he claims his method is applicable to arithmetical functions $a(n)$ which have the representation

$$
a(n)=\sum_{d / n} g(d) h(d, n)
$$

where $g$ and $h$ are arithmetical functions and for each fixed $d, h(d, z)$ is a polynomial in $z$. In fact he illustrated his method (cf. [1], theorems 1, 2, 3 and 4) by considering four well known arithmetical functions.

In $\$ 2$ of this paper we refine Berndt's method so as to be applicable to series of type (1.2) (and more general types also) where the arithmetical function $a(n)$ and the rational function $f$ are arbitrary subject to the only requirement that $\sum f(n)$ and $\sum a(n) f(n)$ are absolutely convergent. In $\S 3$, in addition to deducing theorems 1 through 4 of [1] as special cases of our theorem in $\S 2$, we further illustrate it by choosing particular rational functions while allowing $a(n)$ to be arbitrary. In $\S 4$ we specialize $a(n)$ to evaluate some series, involving the Möbius function $\mu(n)$, the Liouville's function $\lambda(n)$, the Jordan totient function $J(n)$ and the well known Ramanujan's trigonometric sum $c_{n}(m)$, in closed form.

Received by the editors April 25, 1978. 
2. Main result. In the sequel we use the following notation:

Let $a(n)$ be an arithmetical function. For each positive integer $n$ we define $a(-n)$ to be $a(n)$. We denote by $a^{*}(n)$ the unique arithmetical function satisfying $a(n)=\sum_{d \mid n} a^{*}(d)$ or equivalently $a^{*}(n)=\sum_{d \mid n} \mu(d) a(n / d)$ where $\mu$ is the Möbius function. For any set $X$ of positive integers we write $a_{X}(n)$ for $\sum_{d \in X, d \mid n} a^{*}(d)$.

For complex $z$ and positive integral $d$ we write

$$
S(d, z)=\sum_{j=0}^{d-1} \exp (2 \pi i z j / d) \text { and } T(d, z)=\sum_{j=-[(d-1) / 2]}^{[d / 2]} \exp (2 \pi i z j / d)
$$

Clearly for integral $n$

$$
S(d, n)=T(d, n)= \begin{cases}d & \text { if } d \mid n \\ 0 & \text { otherwise }\end{cases}
$$

If $f$ is meromorphic in the complex plane we write $P(=P(f))$ to denote the set consisting of the origin and all the poles of $f$ (when $f$ is rational $P$ is clearly finite). Also $R(f(z): z=\omega)$ stands for the residue of $f$ at $\omega$. We write

$$
s(n)=s_{f}(n)=\sum_{\omega \in \mathbf{P}} R\left(\frac{\pi e^{-\pi i z} S(n, z) f(z)}{\sin \pi z}: z=\omega\right)
$$

and

$$
t(n)=t_{f}(n)=\sum_{\omega \in \mathbf{P}} R\left(\frac{\pi T(n, z) f(z)}{\sin \pi z}: z=\omega\right)
$$

We now prove the following

THEOREM. Let $X$ be a set of positive integers, $a(n)$ an arithmetical function and $f$ a rational function such that $\sum f(n)$ and $\sum a(n) f(n)$ are absolutely convergent. Then

$$
\sum_{\substack{n=-\infty \\ n \notin P}}^{\infty} a_{X}(n) f(n)=-\sum_{n \in X} \frac{a^{*}(n) s(n)}{n}
$$

and

$$
\sum_{\substack{n=-\infty \\ n \notin P}}^{\infty}(-1)^{n} a_{X}(n) f(n)=-\sum_{n \in X} \frac{a^{*}(n) t(n)}{n} .
$$

We require the following

Lemma. Let $a^{\prime}(n)$ denote $\sum_{d \mid n}\left|a^{*}(d)\right|$. Then, under the hypotheses of the theorem, $\sum a^{\prime}(n)|f(n)|$ converges. 
Proof. The hypotheses imply that there is an integer $p \geq 2$ such that $z^{p} f(z)$ tends to a nonzero limit as $z \rightarrow \infty$. Thus it is enough to consider $\sum_{n=1}^{\infty} a^{\prime}(n) n^{-p}$. We have

$$
a^{\prime}(n)=\sum_{r s=n}\left|a^{*}(r)\right| \leq \sum_{r s=n} \sum_{d \delta=r}|a(d)|=\sum_{d \mid n}|a(d)| \tau(n / d)
$$

where $\tau(n)$ denotes the number of positive divisors of $n$. Since $\sum_{n=1}^{\infty}|a(n)| n^{-p}$ and $\sum_{n=1}^{\infty} \tau(n) n^{-p}$ are convergent series of non-negative terms, their Dirichlet product series, namely

$$
\sum_{n=1}^{\infty}\left(\sum_{d \mid n}|a(d)| \tau(n / d)\right) n^{-p},
$$

converges. The lemma now follows.

Proof of the theorem. For each positive integer $m$, we put

$$
A(m, z)=\sum_{d=1}^{m} a^{*}(d) S(d, z) d^{-1}
$$

and

$$
B(m, z)=\sum_{d=1}^{m} a^{*}(d) T(d, z) d^{-1}
$$

If $N$ is a positive integer, let $C_{N}$ denote the positively oriented square with centre at the origin and sides of length $(2 N+1)$ parellel to the real and imaginary axes. For values of $N$ large enough to ensure that $P$ is contained in the interior of $C_{N}$ we consider the integrals

$$
I(m, N)=\frac{1}{2 \pi i} \int_{C_{N}} \frac{\pi e^{-\pi i z} A(m, z) f(z)}{\sin \pi z} d z
$$

and

$$
J(m, N)=\frac{1}{2 \pi i} \int_{C_{N}} \frac{\pi B(m, z) f(z)}{\sin \pi z} d z .
$$

By Cauchy's residue theorem, each of the above integrals equals the sum of the residues of the integrand at its poles in the interior of $C_{N}$. If $n$ is an integer, not in $P$, then

$$
\begin{aligned}
R\left(\frac{\pi e^{-\pi i z} A(m, z) f(z)}{\sin \pi z}: z=n\right) & =A(m, n) f(n) \\
& =f(n) \sum_{\substack{d=1 \\
d \mid n}}^{m} a^{*}(d)
\end{aligned}
$$


in virtue of (2.1). Hence

$$
\begin{aligned}
I(m, N) & =\sum_{\substack{n=-N \\
n \notin P}}^{N} A(m, n) f(n)+\sum_{\omega \in P} R\left(\frac{\pi e^{-\pi i z} A(m, z) f(z)}{\sin \pi z}: z=w\right) \\
& =\sum_{\substack{n=-N \\
n \notin P}}^{N} A(m, n) f(n)+\sum_{d=1}^{m} \frac{a^{*}(d) s(d)}{d} .
\end{aligned}
$$

If $z=x+i y$,

$$
\left|\frac{e^{-\pi i z} A(m, z)}{\sin \pi z}\right| \leq \frac{2 e^{\pi y} \sum_{d=1}^{m}\left|\frac{a^{*}(d)}{d}\right| \sum_{j=0}^{d-1^{\prime}} e^{-2 \pi y j / d}}{\left|e^{\pi y}-e^{-\pi y}\right|}
$$

which is bounded as $y \rightarrow \pm \infty$. Thus there exists a constant $M=M(m)$, independent of $N$, such that for all $z \in C_{N}$,

$$
\left|\frac{e^{-\pi i z} A(m, z)}{\sin \pi z}\right| \leq M
$$

Since the convergence of $\sum|f(n)|$ implies the existence of $a k>0$ and of an integer $c \geq 2$ such that $|f(z)| \leq k|z|^{-c}$ for all large $|z|$, we have

$$
|I(m, N)| \leq 2 M k(2 N+1)\left(N+\frac{1}{2}\right)^{-c} \text {. }
$$

Thus $I(m, N) \rightarrow 0$ as $N \rightarrow \infty$ and from (2.4) we have

$$
\sum_{\substack{n=-\infty \\ n \notin P}}^{\infty} A(m, n) f(n)=-\sum_{d=1}^{m} \frac{a^{*}(d) s(d)}{d} .
$$

Taking limits as $m \rightarrow \infty$ on both sides we obtain

$$
\sum_{\substack{n=-\infty \\ n \in P}}^{\infty} a(n) f(n)=-\sum_{n=1}^{\infty} \frac{a^{*}(n) s(n)}{n},
$$

since on the left side the above lemma allows us to take the limit on $m$ inside the summation sign. Replacing $a^{*}(n)$ by its product with the characteristic function of $X$ one obtains (2.2). The proof of (2.3) follows along the same lines. In particular we obtain

$$
\sum_{\substack{n=-\infty \\ n \in p}}^{\infty}(-1)^{n} a(n) f(n)=-\sum_{n=1}^{\infty} \frac{a^{*}(n) t(n)}{n} .
$$

3. Applications. As mentioned in the introduction we deduce in this section, theorems 1 through 4 of [1] as special cases of the above theorem and record some more results illustrating that theorem. 
For integers $a \geq 0, q \geq 1$ and $(q, a)=1$, put $A(q, a)=\{m q+a \mid m \geq 0, m$ integral\}. Let $\sigma_{\nu}(n), \varphi_{r, s, t}(n), \chi(n), r(n)$ and $\Lambda(n)$ be the arithmetical functions defined by

$$
\sigma_{\nu}(n)=\sum_{d \mid n} d^{\nu}, n^{-t} \varphi_{r, s, t}(n)=\sum_{d \mid n} \mu_{r}^{s}(d) d^{-t}
$$

$\chi(n)=0$ or $(-1)^{(n-1) / 2}$ according as $n$ is even or odd,

$r(n)=4 \sum_{d \mid n} \chi(d)$ (the number of representations of $n$ as a sum of two integral squares) and

$\Lambda(n)=\log p$ or 0 according as $n$ is a power of the prime $p$ or not (known as von Mangoldt's function). Here $r, s, t$ are positive integers with $s \leq 2$ and $\mu_{r}(n)=\mu\left(n^{1 / r}\right)$ or 0 according as $n^{1 / r}$ is an integer or not (known as Klee's generalization of the Möbius function).

Writing $X=A(q, a)$ and taking $a(n)=\sigma_{\nu}(n), r(n)$ and $\log n$ in turn in the theorem of $\S 2$, we obtain theorems 1, 3 and 4 of [1] (noting that $\left.\log n=\sum_{d \mid n} \Lambda(d)\right)$. Further, taking $a(n)=n^{-t} \varphi_{r, s, t}(n)$ and replacing $f(z)$ with $z^{t} f(z)$ in the theorem of $\S 2$, we obtain theorem 2 of [1]. It may be noted that the condition that $f(z)=0\left(|z|^{-c}\right)$ as $|z| \rightarrow \infty$ together with the inequality imposed on $c$ in each of the theorems 1 through 4 of [1] is equivalent to the requirement of absolute convergence of the corresponding series $\sum f(n)$ and $\sum a(n) f(n)$.

Now let $\alpha, \beta, \gamma, \delta$ be complex numbers such that $\alpha \neq n i, \beta \neq n ; \gamma \neq n, \omega^{ \pm 1} n$ and $\delta \neq P^{ \pm 1} n$ for all integers $n$ where $\omega=\exp (2 \pi i / 3)$ and $P=\exp (\pi i / 4)$.

Taking $f(z)=1 /\left(z^{2}+\alpha^{2}\right)$ and adding appropriate residues at $0, i \alpha$ and $-i \alpha$ we obtain

$$
\begin{aligned}
s(n) & =\sum_{j=0}^{n-1}\left\{\frac{1}{\alpha^{2}}+\frac{\pi e^{\pi \alpha(1-2 \mathrm{j} / n)}+\pi e^{-\pi \alpha(1-2 \mathrm{j} / n)}}{2 i \alpha \sin \pi i \alpha}\right\} \\
& =-\frac{n}{\alpha^{2}}\left(\frac{\pi \alpha}{n} \operatorname{coth} \frac{\pi \alpha}{n}-1\right)
\end{aligned}
$$

and

$$
\begin{aligned}
t(n) & =\sum_{j=-[(n-1) / 2]}^{[n / 2]}\left\{\frac{1}{\alpha^{2}}+\frac{\pi e^{-2 \pi \alpha j / n}+\pi e^{2 \pi \alpha j / n}}{2 i \alpha \sin \pi i \alpha}\right\} \\
& =-\frac{n}{\alpha^{2}}\left\{\frac{\pi \alpha}{n} \operatorname{cosech} \frac{\pi \alpha}{n} \cosh \left(\frac{1+(-1)^{n}}{2} \frac{\pi \alpha}{n}\right)-1\right\}
\end{aligned}
$$

by a straight forward calculation. Hence by (2.5) and (2.6) we obtain

$$
\sum_{n=1}^{\infty} \frac{a(n)}{n^{2}+\alpha^{2}}=\frac{1}{2 \alpha^{2}} \sum_{n=1}^{\infty} a^{*}(n)\left(\frac{\pi \alpha}{n} \operatorname{coth} \frac{\pi \alpha}{n}-1\right)
$$


and

$$
\sum_{n=1}^{\infty} \frac{(-1)^{n} a(n)}{n^{2}+\alpha^{2}}=\frac{1}{2 \alpha^{2}} \sum_{n=1}^{\infty} a^{*}(n)\left(\frac{\pi \alpha}{n} c h_{n}(\pi \alpha)-1\right)
$$

where $c h_{n}(\theta)=\operatorname{coth} \theta / n$ or $\operatorname{cosech} \theta / n$ according as $n$ is even or odd.

Taking

$$
f(z)=\frac{1}{(z+\beta)^{2}}, \frac{1}{z^{3}+\gamma^{3}} \text { and } \frac{1}{z^{4}+\delta^{4}}
$$

in turn and proceeding as above, we obtain

$$
\begin{aligned}
& \sum_{\substack{n=-\infty \\
n \neq 0}}^{\infty} \frac{a(n)}{(n+\beta)^{2}}=\frac{1}{\beta^{2}} \sum_{n=1}^{\infty} a^{*}(n)\left\{\left(\frac{\pi \beta}{n} \operatorname{cosec} \frac{\pi \beta}{n}\right)^{2}-1\right\}, \\
& \sum_{\substack{n=-\infty \\
n \neq 0}}^{\infty} \frac{(-1)^{n} a(n)}{(n+\beta)^{2}}=\frac{1}{\beta^{2}} \sum_{n=1}^{\infty} a^{*}(n)\left\{\left(\frac{\pi \beta}{n} \operatorname{cosec} \frac{\pi \beta}{n}\right)^{2} \cos \left(\frac{1-(-1)^{n}}{2} \frac{\pi \beta}{n}\right)-1\right\}, \\
& \sum_{\substack{n=-\infty \\
n \neq 0}}^{\infty} \frac{a(n)}{n^{3}+\gamma^{3}}=\frac{1}{3 \gamma^{3}} \sum_{n=1}^{\infty} a^{*}(n)\left\{\frac{\pi \gamma}{n} \cot \frac{\pi \gamma}{n}\right. \\
& \left.\quad+\frac{\pi \gamma \omega}{n} \cot \frac{\pi \gamma \omega}{n}+\frac{\pi \gamma \bar{\omega}}{n} \cot \frac{\pi \gamma \bar{\omega}}{n}-3\right\}, \\
& \sum_{\substack{n=-\infty \\
n \neq 0}}^{\infty} \frac{(-1)^{n} a(n)}{n^{3}+\gamma^{3}}=\frac{1}{3 \gamma^{3}} \sum_{n=1}^{\infty} a^{*}(n)\left\{\frac{\pi \gamma}{n} c_{n}(\pi \gamma)\right. \\
& \left.\quad+\frac{\pi \gamma \omega}{n} c_{n}(\pi \gamma \omega)+\frac{\pi \gamma \bar{\omega}}{n} c_{n}(\pi \gamma \bar{\omega})-3\right\}
\end{aligned}
$$

where $c_{n}(\theta)=\cot \theta / n$ or $\operatorname{cosec} \theta / n$ according as $n$ is even or odd,

$$
\sum_{n=1}^{\infty} \frac{a(n)}{n^{4}+\delta^{4}}=\frac{1}{4 \delta^{4}} \sum_{n=1}^{\infty} a^{*}(n)\left\{\frac{\pi \delta \rho}{n} \cot \frac{\pi \delta \rho}{n}+\frac{\pi \delta \bar{\rho}}{n} \cot \frac{\pi \delta \bar{\rho}}{n}-2\right\}
$$

and

$$
\sum_{n=1}^{\infty} \frac{(-1)^{n} a(n)}{n^{4}+\delta^{4}}=\frac{1}{4 \delta^{4}} \sum_{n=1}^{\infty} a^{*}(n)\left\{\frac{\pi \delta \rho}{n} c_{n}(\pi \delta \rho)+\frac{\pi \delta \bar{\rho}}{n} c_{n}(\pi \delta \bar{\rho})-2\right\}
$$

4. Evaluation of some series. In this section, by suitably specializing $a(n)$, we deduce interesting formulae from the theorem of $\S 2$ as well as from the identities listed in $\S 3$.

If $a^{*}(n)$ is the characteristic function of $\{x\}, x$ a positive integer, we see that $a(n)$ turns out to be the characteristic function of the set of all non-zero 
multiples of $x$ so that, by (2.5) and (2.6), we have

$$
\sum_{n \neq 0, n=0(\bmod x)} f(n)=-s(x) / x
$$

and

$$
\sum_{n \neq 0, n=0(\bmod x)}(-1)^{n} f(n)=-t(x) / x
$$

If $a^{*}(n)$ is an arithmetical function vanishing outside a finite set then $a^{*}(n)$ is a (complex) linear combination of characteristic functions of singletons so that, in this case, one easily obtains formulae analogous to (4.1) and (4.1').

If $a(n)$ is the characteristic function of a set $X$ of positive integers, the corresponding function $a^{*}(n)$, usually called the inversion function of the set $X$, is denoted by $\mu_{X}(n)$. Formulae (3.1) through $\left(3.4^{\prime}\right)$ may be specialized for this characteristic function. For example, one can write, in virtue of (3.1),

$$
\sum_{ \pm n \in x} \frac{1}{n^{2}+\alpha^{2}}=\frac{1}{\alpha^{2}} \sum_{n=1}^{\infty} \mu_{X}(n)\left(\frac{\pi \alpha}{n} \operatorname{coth} \frac{\pi \alpha}{n}-1\right) .
$$

Taking $X=\{1\}$ we see that $\mu_{X}(n)=\mu(n)$ so that (4.2) yields

$$
\sum_{n=1}^{\infty} \mu(n)\left(\frac{\pi \alpha}{n} \operatorname{coth} \frac{\pi \alpha}{n}-1\right)=\frac{2 \alpha^{2}}{\alpha^{2}+1} .
$$

If $X$ is the set of all positive integers that are prime to a fixed positive integer $m$, one can easily verify that $\mu_{X}(n)=\mu(n)$ or 0 according as $n \mid m$ or not. Hence by (4.2) one has

$$
\sum_{\substack{n=1 \\(n, m)=1}}^{\infty} \frac{1}{n^{2}+\alpha^{2}}=\frac{1}{2 \alpha^{2}} \sum_{n \mid m} \mu(n)\left(\frac{\pi \alpha}{n} \operatorname{coth} \frac{\pi \alpha}{n}-1\right) .
$$

Taking $X$ to the set of all integral squares we see that $\mu_{X}(n)=\lambda(n)=$ $(-1)^{\Omega(n)}(\lambda$ is known as the Liouville's function) where $\Omega(n)$ is the number of prime factors of $n$, counting repetitions. Now replacing $\alpha$ by $\delta^{2}$ in (4.2) we obtain

$$
\begin{aligned}
\sum_{n=1}^{\infty} \lambda(n)\left(\frac{\pi \delta^{2}}{n} \operatorname{coth} \frac{\pi \delta^{2}}{n}-1\right) & =2 \delta^{4} \sum_{n=1}^{\infty} \frac{1}{n^{4}+\delta^{4}} \\
& =\frac{1}{2}(\pi \delta \rho \cot \pi \delta \rho+\pi \delta \bar{\rho} \cot \pi \delta \bar{\rho})-1
\end{aligned}
$$

Here we made use of (3.4) with $a(n)=1$ for all $n$ (so that $a^{*}(n)=1$ or 0 according as $n=1$ or $n>1)$.

Taking $a^{*}(n)=J(n)=\varphi_{1,1,2}(n)$ (see $\S 3$ above), known as Jordan's totient 
function of order 2, we note that $a(n)=n^{2}$. Now (3.4) yields

$$
\begin{aligned}
& \sum_{n=1}^{\infty} J(n)\left(\frac{\pi \delta \rho}{n} \cot \frac{\pi \delta \rho}{n}+\frac{\pi \delta \bar{\rho}}{n} \cot \frac{\pi \delta \bar{\rho}}{n}-2\right) \\
&=4 \delta^{4} \sum_{n=1}^{\infty} \frac{n^{2}}{n^{4}+\delta^{4}}=\pi \sqrt{2} \delta^{3} \frac{\sinh \pi \delta \sqrt{2}-\sin \pi \delta \sqrt{2}}{\cosh \pi \delta \sqrt{2}-\cos \pi \delta \sqrt{2}}
\end{aligned}
$$

The last step is obtained by writing $n^{2} /\left(n^{4}+\delta^{4}\right)=\frac{1}{2}\left(1 /\left(n^{2}+\rho^{2} \delta^{2}\right)+\right.$ $1 /\left(n^{2}+\bar{p}^{2} \delta^{2}\right)$ and applying (3.1) separately with $a(n)=1$ for all $n$.

Concerning the well known Ramanujan's trigonometric sum $c_{n}(m)$, defined to be the sum of the $m$ th powers of the primitive $n$th roots of unity, it is known that $\sum_{d / n} c_{d}(m)=n$ or 0 according as $n \mid m$ or not (cf. [2], §1.5). Hence, taking $a^{*}(n)$ to be $c_{n}(m)$ where $m$ is a fixed positive integer in (3.1), we obtain

$$
\sum_{n=1}^{\infty} c_{n}(m)\left(\frac{\pi \alpha}{n} \operatorname{coth} \frac{\pi \alpha}{n}-1\right)=2 \alpha^{2} \sum_{n \mid m} \frac{n}{n^{2}+\alpha^{2}}
$$

Incidentally we note, since $c_{n}(1)=\mu(n)$, that (4.3) is a special case of (4.7).

Dividing both sides of (4.7) by $\alpha^{2}$ and letting $\alpha \rightarrow 0$, one obtains

$$
\sum_{n=1}^{\infty} \frac{c_{n}(m)}{n^{2}}=\frac{6}{\pi^{2}} \sigma_{-1}(m)
$$

Working similarly with results analogous to (4.7) that may be obtained from (3.1'), (3.4) and $\left(3.4^{\prime}\right)$ respectively, we get

$$
\begin{gathered}
\sum_{n=1}^{\infty} \frac{(-1)^{n} c_{n}(m)}{n^{2}}=\frac{2}{\pi^{2}}\left(4 E_{-1}(m)-\sigma_{-1}(m)\right), \\
\sum_{n=1}^{\infty} \frac{c_{n}(m)}{n^{4}}=\frac{90}{\pi^{4}} \sigma_{-3}(m)
\end{gathered}
$$

and

$$
\sum_{n=1}^{\infty} \frac{(-1)^{n} c_{n}(m)}{n^{4}}=\frac{6}{\pi^{4}}\left(16 E_{-3}(m)-\sigma_{-3}(m)\right)
$$

where $E_{v}(m)$ stands for $\sum_{n \mid m}(-1)^{n} n^{\nu}$. Where as (4.8) and (4.9) are due to S. Ramanujan (cf. [2], §1.5), $\left(4.8^{\prime}\right)$ and $\left(4.9^{\prime}\right)$ are believed to be new. 


\section{REFERENCES}

1. Bruce C. Berndt, A new method in arithmetical functions and contour integration, Canad. Math. Bull, 16 (1973), 381-388.

2. E. C. Titchmarsh, The Theory of the Riemann Zeta function, Oxford University Press, 1967.

Department of Mathematics

ANDHRA UNIVERSTTY

WALTAIR, INDIA 530003 